\title{
Towards the Hybrid Library: Developments in UK Higher Education
}

\author{
Chris Rusbridge \\ Director of Information Services, University of Glasgow \\ C.Rusbridge@compserv.gla.ac.uk
}

Prof. Bruce Royan

CEO, Scottish Cultural Resources Access Network

bruce@scran.ac.uk

\begin{abstract}
The real world in which information professionals struggle to provide high quality services is not the simple world of most so-called "digital library" services, but rather is characterised by complexity and diversity in almost all aspects of the information access chain. Dealing with diversity is the real problem for providers interested in offering quality services, and for users seeking to access relevant sources to answer their information problems.

This paper outlines the efforts of the UK Joint Information Systems Committee (JISC) to help UK Higher Education Institutions deal with this growing diversity of information resources. These efforts are based on two strands: the Electronic Libraries Program and the development of the JISC Collections. These strands are now coming together as JISC concentrates on developing a Distributed National Electronic Resource (DNER), and encourages organisations to harmonise and facilitate access to this and a plethora of other resources, digital and conventional, through the model of the Hybrid Library.
\end{abstract}

This paper contains material presented at the VALA Conference in Melbourne Australia, in February 2000. 


\section{Introduction}

The term "Hybrid Library" conjures up an image of some triumph of Genetic Engineering; part bookhouse, part scorpion, with a dash of eye of newt. To continue the metaphor, it describes a specie of library adaptive to today's increasingly complex and turbulent information environment. The term is not necessarily synonymous with Organizational Convergence, since it describes a process of service provision, rather than administrative structure. Less elegant perhaps than its US equivalent the "Gateway Library", it nevertheless conveys a Janus-headed service driven by a recognition that despite the burgeoning of the internet and digital publication, the vast majority of useful information resources in academic libraries are and will remain print based. The Hybrid Library, by exploiting both access and storage, Clicks and Mortar, seeks to provide the end-user with, in Ian Winkworth's phrase, "a managed blend of traditional and electronic resources".

\section{Follett report}

The development of Hybrid Libraries in the UK had a somewhat unlikely start. The abolition in 1992 of the "binary divide" between the older universities and the polytechnics approximately doubled the number of universities. Library provision in the former polytechnics had been chronically under-funded, and there was serious concern at the potential impact of having to upgrade all these libraries to "research quality".

This issue was the genesis for the Joint Funding Councils' Libraries Review Group, which in November 1993 produced its findings in the "Follett report", as it is colloquially referred to after the Chair of the Committee, Prof. Sir Brian Follett. This Report was one of the most influential of recent years, if measured by the amount of spending on its recommendations. Chapter 7 of the Report related to the use of IT to alleviate library problems. The implementation of this part of the Report was delegated to the HE Funding Councils' Joint Information Systems Committee (JISC), with a budget of $£ 15$ million over 3 years. Within JISC, this implementation was handed to a newly created sub-committee, the Follett Implementation Group for IT, with the splendid acronym of FIGIT.

\section{eLib Phases 1 and 2}

FIGIT's response to the agenda laid out in Chapter 7 of the Follett report was to call for proposals for what became the Electronic Libraries Program, or eLib. Two calls were made, and the resulting Phases 1 and 2 of eLib comprised almost 60 projects http://www.ukoln.ac.uk/services/elib/

It is impossible to sum up the results of 60 projects in a few sentences, but a few points are worth noting here in view of later developments:

A low technology, distributed document delivery co-operative was set up (LAMBDA), providing both price and performance competition with British Library's Document Supply Centre, without however challenging the latter's entrenched position. Our ambitions for user-initiated document delivery 
remain un-realised as yet due to a variety of factors including delays in software delivery.

< Non-destructive digitisation is extremely expensive, particularly for older material (especially pre-19th century). It is not easy to justify on space-saving grounds, although it can be eminently justifiable in terms of accessibility. Copyright material, particularly when including many pictorial images, remains a serious problem.

Providing extracts of key texts on demand in print or especially electronic form is a valuable support for learners. Publishers began to understand and even accept this practice, and some economic factors are better understood. However, without support in copyright law for fair use in this area, copyright clearance and then digitisation activities (especially when OCR is used to convert to text, because of the proof-reading overhead) introduced such delays into a time-critical process, that the system is unlikely to work for institutions acting alone.

$<$ The change towards producing parallel print and digital versions of journals increases costs in the short term. While new journals with no print equivalent can be created, those which make full and effective use of the new medium (eg Internet Archaeology) are also very expensive. Meanwhile the economic models for freely accessible electronic journals remain unclear, while the technology for subscription-based electronic journals is much more intrusive than in the print world.

< Librarians respond extremely positively to the pressure for change, driven by a strong service ethic. Some academics also grasp the opportunities for change, but careful co-ordination with the academic cycle is essential (and often difficult to achieve). Many academics do not have the time to experiment in their use of technological change. Some academics are distinctly technophobic, at least in their teaching practice. Cultural change by retirement may be an important factor!

< Dissemination is therefore a major issue, and one that is too often insufficiently stressed. If the goals of a program include cultural change in a community, it is not enough to report on results via web pages, conference papers or journal articles. There needs to be a sustained dissemination program.

The comments above are reflections of the current authors, rather than the results of the independent summative evaluation of eLib recently completed. Though they appear somewhat negative, we believe the program had enormous impact in changing the direction of library provision towards the digital domain, including:

$<$ A sea-change in attitudes in and towards the LIS community.

$<$ A change in direction for JISC from being a network provider with a bit of information to a realisation that it is committed to the information enabling business. 


\section{eLib phase 3 and the Hybrid Library}

When contemplating what should come after the first 2 phases of eLib, it was time to think beyond the bounds of the Follett report. Phase 3 was concerned with converting successful Elib projects into self-sustaining services, building a distributed national union catalogue, working towards a national policy for digital preservation, and in particular, exploring the feasibility of the Hybrid Library. As explained above, the motivation behind the hybrid library program area was a need to cope with diversity. Diversity is a major problem as real libraries struggle to come to grips with the digital information world:

$<$ Results from eLib Phase 1/2 projects, and from other programs internationally, were extremely varied, but there had been little study of the impacts of bringing in several of these technologies to play in real library environments.

As a corollary to the above, many "digital library" projects (especially those from the US National Science Foundation's Digital Libraries Initiative) had been expressed in terms quite independent from real library environments. Digital Library projects often appear to be "single topic" services without the needed breadth. We felt libraries had a continuing value in HEIs, whether or not the domain was print or digital. In particular, libraries have roles in selection, presentation and mediation of resources, although they deal with them in very format-specific ways. So diversity already exists within the library; one view of the library is as imposer of order on diversity. Even for existing or legacy digital material, mostly CD-ROMs and bibliographic or fulltext datasets, the interfaces which are offered are extremely varied, not to say idiosyncratic; specialisation and differentiation of interfaces have occurred as vendor marketing tools. The result is a hodgepodge of different approaches which the would-be user of information must navigate. In truth these different approaches are barriers to the user; they are sustainable only while there are small numbers of digital resources but will not be as these numbers increase.

The idea of the hybrid library program area developed from these and related thoughts. As is usual, the final program to emerge from the proposals presented in response to the call may not have explored these areas as deeply in some areas as we would have liked. Nevertheless the program outlined below is producing some very interesting results, which indicate that much can be achieved with some careful thought and modest investment.

\section{Agora}

Agora is working with a commercial vendor to develop a standards-based broker system (based on a 3-tier architecture with thin, web-based client, intelligent brokers based around library policies, and distributed resource providers) suitable for hybrid library use. The architecture is based on the MODELS Information Architecture (Gardner, Miller and Russell). The broker aims to provide levels of integration across diverse data sets mainly through the use of Z39.50, and expects to integrate more than 40 Z39.50-based resources. This project has been adversely affected by difficulties experienced by their commercial partner. 
Agora supports the aggregation of resources in groups called "information landscapes" which can then be searched. The same idea appears again in HeadLine, below, and Agora has worked with other Phase 3 projects to develop collection level descriptions (Brack), to help define the information landscape. Agora provides a complete process for the user from discovery of a collection through to a document request and delivery.

\section{BUILDER}

BUILDER is working in an institutional context, and aims to exploit all the synergies possible in the institutional resources available to them, to deliver innovative services.

Although BUILDER appears to have focused on products, this is because of its belief that demonstration is better than explanation. Their cycle could be described as "think far, build near, try out and evaluate." Much of this work has centred on toolkits for their particular local environment: Talis for the library management system, and IIS with SiteServer for the web server. These tools are linked together in clever ways to produce a whole variety of demonstrator products which can be viewed on their web site.

Probably the most popular service is the exam paper service, which has been formally evaluated (Dalton and Nankivell). It was initially restricted to on-campus access for legal reasons, but this year being extended off campus with added authentication. To this end they have explored authentication approaches linked to their Novell LAN and also to their particular OPAC's borrower identification system. They have also looked at the integration of electronic journals, and of local and remotely digitised resources. They have run a pilot electronic short loan system involving over 60 documents including 4 complete books; once again this has been formally evaluated (Dalton and Nankivell).

\section{HeadLine}

"The information landscape" is a term used to refer to the set of information resources of interest at any one time to a user. HeadLine is particularly concerned with tailoring information landscapes. To this end HeadLine is constructing an interface based around a Personal Information Environment (PIE) which allows groups of users to be presented with initial views from their teachers but subsequently to adapt these to suit their own needs. Authentication and authorisation are critical for this work, as are links to MIS systems so that the initial requirements of students can be assessed automatically. Building these links has been found to be considerably more complex than was expected.

The project has also completed a significant analysis of library information service enquiries, and has prototyped a system called SHERLOC to help users find documents on the physical shelves (Shelfmark \& Resource Locator). They are investigating a document delivery service between the partner sites, of the kind useful to a multicampus institution. 


\section{HYLIFE}

HYLIFE is interesting in demonstrating the wide variety of solutions which may be appropriate for different groups of users. It is our most "geographically challenged" project, including Plymouth in the south and the University of the Highlands and Islands Project in the far north, with several partners in between. Some aspects of the project are already being brought into service at the University of Northumbria at Newcastle.

Interesting findings from HYLIFE include evidence that students view information retrieved electronically as intrinsically more valid than print sources. Given widespread concern in the LIS community at the difficulty in distinguishing garbage from good digital information, this emphasis emphasises the need for guidance on quality.

The HYLIFE annual report for 1999 also raises concerns at issues related to what it calls "the convergence of book and gown" (chapter 3). It is getting less possible to clearly distinguish and separate the educational, academic process, managed by faculty, from information provision, managed by the library. Information delivery becomes an intimate part of the educational process. Although HYLIFE is concerned at a perceived threat to funding independence for the library, there is also clearly value in being an increasingly irreplaceable part of the whole process.

\section{MALIBU}

MALIBU has also made progress on many fronts, but two in particular are worth noting. The first is a pre-prototype searching agent allowing cross searching of web sites using HTTP (sometimes disparagingly referred to as HTML scraping) (Harris). The advantage of this implementation over rivals is claimed to be the ability to bypass the target's state while maintaining its own state as a broadcast search. Although it is potentially high maintenance, this approach may prove extremely valuable in the short to medium term.

The other major development in MALIBU is the pair of complementary models of the Hybrid Library (Wissenburg). The first is a user model and the second is a technical services model. Forming models of the hybrid library was one of the tasks for the projects.

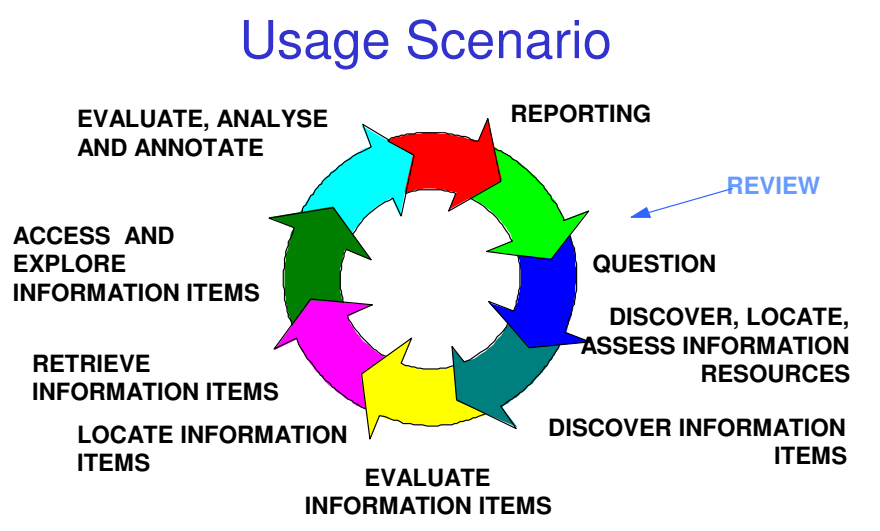

Figure 1: MALIBU Usage Scenario model 
The user model shows the stages a user goes through, often iteratively, in discovering, evaluating and using information. The model above starts from the user having some kind of question.

\section{Technical systems}

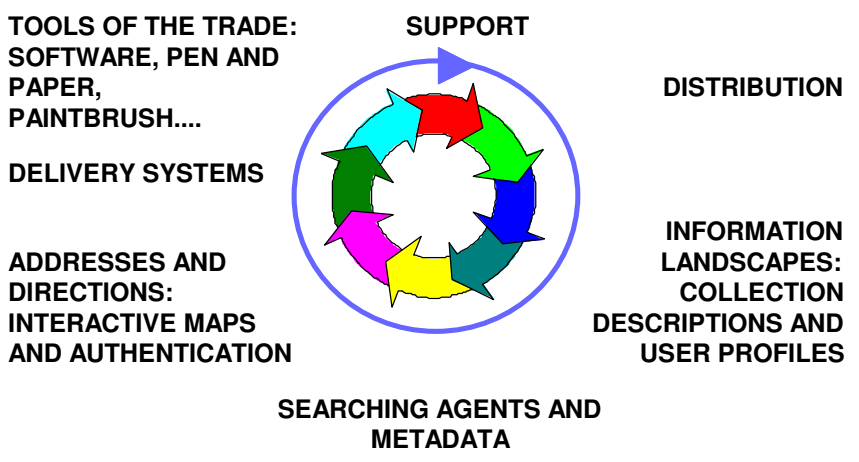

Figure 2: MALIBU Technical Systems model

The technical services model shows the services that are needed to support these user stages. See the MALIBU documentation for further ideas on the applicability of these models. The key here is the extent to which the use of information has to be closely linked to - or embedded in - the delivery of the information.

The hybrid library seems to have been an idea whose time had come; in addition to the eLib projects reported on above, several unsuccessful bidders nevertheless decided to continue with their hybrid library plans, albeit on a reduced scale. And all the hybrid library work in the UK has proceeded in step with, and informed by, JISC-funded contributions to nationwide electronic resource discovery

\section{The Resource Discovery Network}

One of the successes of eLib Phase 1 was the set of subject-based Internet gateways (eg ADAM, EEVL, OMNI, SOSIG etc), which provided quality-tested access to collections of Internet-based resources. This idea was worth extending, but it was not easy to see how this could occur fairly across the subject spectrum. The decision was taken to establish a networked organisation, the Resource Discovery Network (RDN), which would integrate and extend this work, seeking additional financial and other support.

The RDN is organisationally based on the model tested with the Arts and Humanities Data Service, with the RDN Centre running common services, interoperability standards and systems. A range of "faculty-level" hubs addressing a larger subset of the subject spectrum are located in institutions with strong links to the subjects embraced by the hub; this subject-linkage is seen as one of the strengths of the approach. Each faculty hub may have a number of subject-level gateways associated with it. Initial hubs have been created based on eLib projects covering social sciences, business and law; engineering, maths and computing; and medical/biomedical. Additional hubs are being established covering humanities and physical sciences. At least 3 more hubs are needed, but the funding is hard to find. 


\section{The JISC Collections}

Meanwhile, JISC was continuing to develop its portfolio of digital collections. Initially, these had been presented to users through home grown and proprietary interfaces. The prime example of this was the ISI Citation Service, delivered by Bath Information and Data Services (BIDS). Later services began to develop from this base, providing a family resemblance for users. Services were established at 3 Data Centres. Then, as always, the limitations of proprietary in house developments began to emerge, and there was pressure to use commercial interfaces which the student might encounter later in the real world. This extended to the point where one dataset (INSPEC) was offered with a choice of interfaces from 6 data hosts, a separate choice by the library once the decision to subscribe was taken. While this approach gave some benefits, it started to increase the diversity problems already referred to.

Now the collection extends to over 40 datasets covering areas such as statistical and geospatial data as well as bibliographic and full text.

An important development has been the development of a JISC "Collections Policy" (An integrated information environment for higher education), describing the framework in which collection and retention decisions will be made.

\section{The DNER}

The Distributed National Electronic Resource was initially the expression of two simple ideas. First was the notion that the provision of digital resources should be physically distributed for redundancy and avoidance of single points of failure. Second was the belief that the collections offered should fit within a national framework, the JISC Collections Policy.

Over time this approach to the DNER began to develop, spurred by the increasing diversity of the resources being offered and by concerns about the sustainability of this diversity:

The location of resources was determined more by historical "accidents of negotiation" than by logic (at least as far as the user could determine). This was in keeping with the distributed idea, but it turns out that different Data Centres have their own differentiation (more diversity). Also, it appears that users have a greater sense of "network place" than we had expected.

< The diversity of interfaces has already been noted. It is not so much the diversity itself (since fitness for purpose will always drive some diversity), but the wanton use of diversity as a market differentiation tool, which is of concern. We believe in different interfaces, oriented to the needs of particular user groups.

There was beginning to be a diversity of authentication approaches. As the idea of the DNER moved in concept from a small set of individual resources towards resources as components of a whole, the problem of authentication and authorisation was thrown into sharp relief. Bluntly, users did not want to remember more usernames and passwords. The response to this was ATHENS 
3, about which little more can be written here, other than that it is very valuable, far from perfect, possibly inadequate for the task, a triumph of pragmatism, and/or a disaster in the making. Take your pick!

\ There was an increasing need to be able to "join up" different services, so that when a bibliographic reference is discovered from a search of an abstracting and indexing dataset, the location of the journal could be discovered from a union catalogue, and the article requested via ILL or document delivery. This joined up integration was impossible with the diversity of interfaces. A dataset independent protocol such as Z39.50 appeared potentially a most important component.

Any particular user group will have interests in a range of datasets from different data providers. The DNER allows a user group to provide access to this range of resources, independent of the data provider, in much the way that a library's books are arranged by subject but not by publisher.

The DNER plans the construction of portals to facilitate user-centred access to the resources. Portals are envisaged to be standards-based web-fronted brokers (probably using Z39.50 and other appropriate protocols), similar to the hybrid library broker in Agora, capable of multiple types of integration. This integration would include:

a) Integration of access to existing services, through a variety of entry points tailored to appropriate communities rather than to the data owners, data suppliers or even data types.

b) Integration through enabled cross-searching; the ability in one search to access several datasets (we call this breadth rather than depth searching, as only the common data features will be searchable and some of the functionality will be lost).

c) Integration through linking to value-added services such as ILL, document acquisition transactions, etc, especially in a "joined-up" way where information is carried across appropriately and does not have to be re-keyed.

d) Integration across domains, eg searching across different media types, curatorial traditions etc.

e) Access to a wide range of sources through non-traditional interfaces.

So we have again a 3-layer architecture: a set of resources at the bottom, a set of portals based on brokers in the middle, and the users through web browsers at the top. There would be many cross-linkages between the layers.

The set of portals might include:

< One central, JISC portal: a starting place for anyone, especially those who have not yet identified a specialist portal which suits their needs.

$<$ A set of subject-oriented portals; these are seen as natural extensions of the RDN faculty-level hubs and their associated subject gateways. 
An extension of the hybrid library idea to encompass local portals to the DNER. Local portals could support access to non-JISC resources licensed by the institution. A local portal could even be extended as "personal portals", including access to resources which an individual has subscribed to.

< More specialised portals further into the future. First and simplest of these could be portals dedicated to particular media types such as still images, and time-based media such as movies or sound, or maps.

$<$ Portals with specific world views, such as a geo-spatial portal.

Out of this will emerge the idea of different views of the same data appropriate to different groups of users.

\section{Conclusion}

To conclude, the eLib program has developed from a diverse set of projects in Phases 1 and 2 to a rather more focused set in Phase 3, in which the hybrid library projects represent a particularly important strand. The idea of the DNER has developed from simple beginnings to a complex concept of "joined up services". Underlying infrastructure issues including access management, middle-ware and standards have been tackled. A significant portfolio of datasets has been amassed. The future holds increasing attempts to control the increasing diversity by coupling national provision of a DNER, with an institutional emphasis on making digital resources more accessible for learning and teaching.

\section{Works and projects cited}

AGORA. 1997. University of East Anglia. Available: http://hosted.ukoln.ac.uk/agora/

Arts and Humanities Data Service. 1995. Kings College, London. Available: http://www.ahds.ac.uk/

ATHENS Access management. 1997. NISS. Available: http://www.athens.ac.uk/

Brack, Verity. 1999. e-Lib Collection Description Scheme, ver 1.0. University of Sheffield. Available http://www.shef.ac.uk/ riding/cld/cldschem.html

BUILDER. 1997. University of Birmingham. Available: http://builder.bham.ac.uk/

Dalton, Peter and Nankivell, Clare. 1999 The University of Birmingham Exam Paper Database: an analysis of user accesses and feedback from 19 December 1998 to 25 June 1999. University of Central England. Available http://builder.bham.ac.uk/reports/pdf/exam.pdf.

Dalton, Peter and Nankivell, Clare. 1999. The University of Birmingham Pilot Electronic Short Loan Service: an analysis of user access logs between 1 January 1999 and 30 June 1999. University of Central England. Available http://builder.bham.ac.uk/reports/pdf/esllog.pdf. 
Dalton, Peter and Nankivell, Clare. 1999. Analysis of the questionnaire survey concerning the use of the University of Birmingham Pilot Electronic Short Loan Service. University of Central England. Available http://builder.bham.ac.uk/reports/pdf/eslquestionnaire.pdf.

CEDARS. 1998. Consortium of University Research Libraries. Available: http://www.curl.ac.uk/projects.shtml

CLA and the Digitisation of Text. 1999. Copyright Licensing Agency. Available http://www.cla.co.uk/www/digital.htm.

COPAC. 1996. Consortium of University Research Libraries. Available: http://www.copac.ac.uk/copac/

The DNER: Description of the DNER. 1999. Joint Information Systems Committee. Available: http://www.jisc.ac.uk/pub99/dner_desc.html

eLib: Electronic Libraries Programme. 1995. UK Office for Library and Information Networking. Available: http://www.ukoln.ac.uk/services/elib/

Gardner, Tracy with Miller, Paul and Russell, Rosemary. The MIA Logical Architecture. Draft 0.3. 1999. UK Office for Library and Information Networking. Available: http://www.ukoln.ac.uk/dlis/models/requirements/arch/

Harris, Stephen. The MALIBU Pre-prototype: broadcast searching disparate HTTP targets. 1999. Kings College, London. Available http://www.kcl.ac.uk/humanities/cch/malibu/reports/searhttp.htm.

HeadLine. 1997. London School of Economics. Available: http://www.headline.ac.uk/

The HeadLine Personal Information Environment (PIE). 1999. London School of Economics. Available http://www.headline.ac.uk/public/diss/nl-pie.pdf.

HERON. 1998. University of Stirling. Available: http://www.stir.ac.uk/infoserv/heron/

HYLIFE. 1997. University of Northumbria at Newcastle. Available: http://www.unn.ac.uk/ xcu2/hylife/

HYLIFE Annual report. 1999. University of Northumbria at Newcastle. Available http://www.unn.ac.uk/ xcu2/hylife/Annrephome.html.

Internet Archaeology. 1996. Council for British Archaeology. Available: http://intarch.ac.uk/

JISC Circular 5/99. 1999. Joint Information Systems Committee. Available: http://www.jisc.ac.uk/pub99/c05_99.html

Imesh Toolkit. 1999. UK Office for Library and Information Networking. Available http://www.imesh.org/toolkit/.

An integrated information environment for higher education: developing the Distributed, National Electronic Resource (DNER). 1997. Joint Information Systems Committee. Available: http://www.jisc.ac.uk/cei/dner_colpol.html

Joint Funding Councils’ Libraries Review Group. Report. Bristol: HEFCE, 1993. 
Lunau, Carroll; Miller, Paul and Moen, William E. (Eds.). The Bath Profile: An International Z39.50 Specification for Library Applications and Resource Discovery (draft). 1999. UK Office for Library and Information Networking. Available: http://www.ukoln.ac.uk/interopfocus/activities/z3950/int_profile/bath/draft/stable1.html

MALIBU. 1997. Kings College, London. Available: http://www.kcl.ac.uk/humanities/cch/malibu/

National Electronic Site Licence Initiative. 1998. University of Manchester. Available http://www.nesli.ac.uk/

Newton-Ingham, Greg with Palmer, David, Kay, David and Smith, Neil. 1999. Agora Hybrid Library Management System (HLMS) requirements catalogue: release 1 specification. University of East Anglia. Available http://hosted.ukoln.ac.uk/agora/documents/agora_spec_public1.doc

Powell, Andy. The Resource Discovery Network (RDN) architecture. 1999. UK Office for Library and Information Networking. Available http://www.ukoln.ac.uk/dlis/models/models9/presentations/rdn.ppt

Resource Discovery Network. 1999. Kings College, London. Available: http://www.ukoln.ac.uk/dlis/models/models9/presentations/rdn.ppt

Shelfmark \& Resource Locator (SHERLOC). 1999. London School of Economics. Available http://decomate.lse.ac.uk/john/cgi-bin/sherloc/sherloc.cgi.

Wissenburg, Astrid. MALIBU: Hybrid Library Models version 1. 1999. Kings College, London. Available http://www.kcl.ac.uk/humanities/cch/malibu/reports/modv1.htm 